Provided for non-commercial research and education use. Not for reproduction, distribution or commercial use.

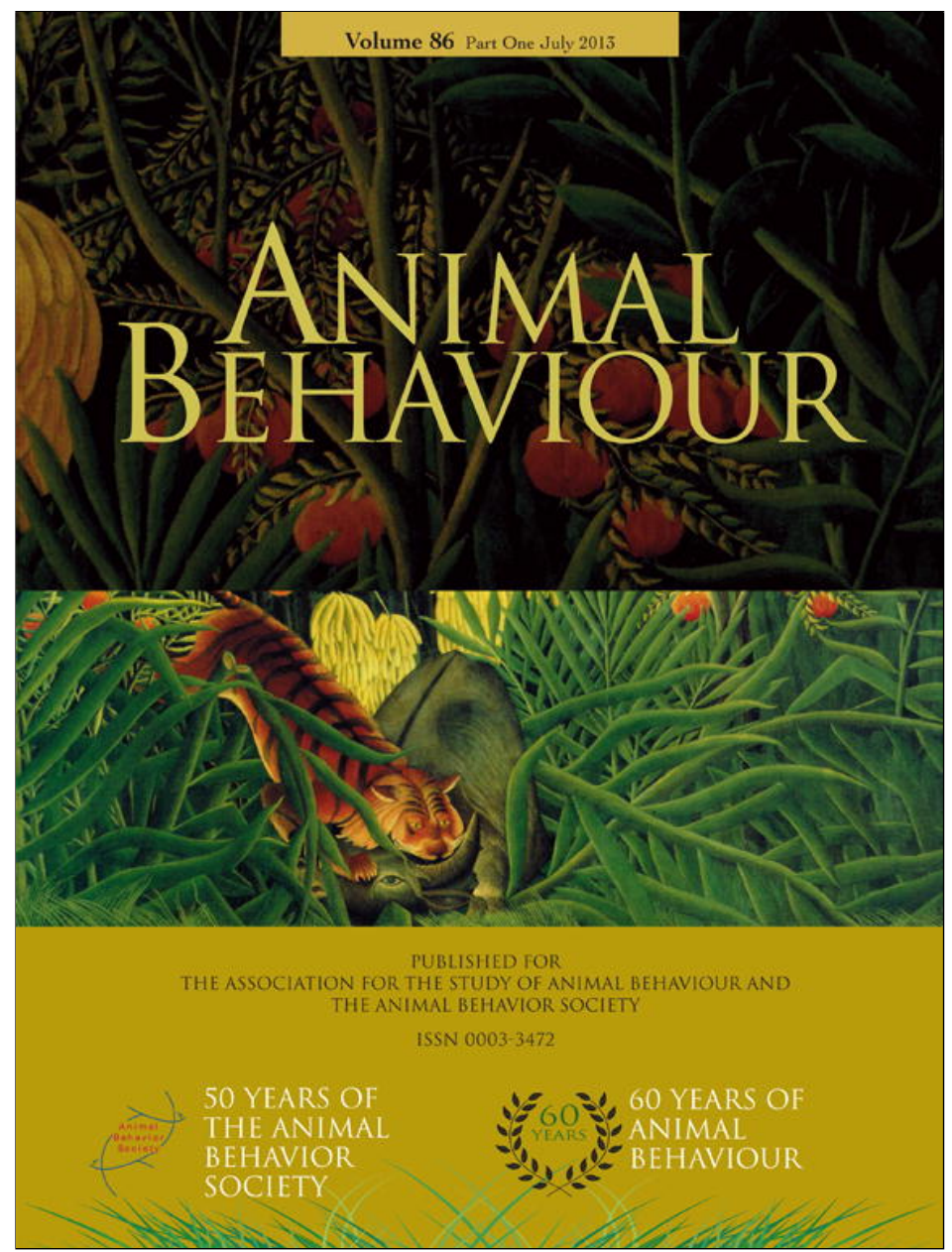

This article appeared in a journal published by Elsevier. The attached copy is furnished to the author for internal non-commercial research and education use, including for instruction at the authors institution and sharing with colleagues.

Other uses, including reproduction and distribution, or selling or licensing copies, or posting to personal, institutional or third party websites are prohibited.

In most cases authors are permitted to post their version of the article (e.g. in Word or Tex form) to their personal website or institutional repository. Authors requiring further information regarding Elsevier's archiving and manuscript policies are encouraged to visit:

http://www.elsevier.com/authorsrights 


\title{
Barn owls do not interrupt their siblings
}

\author{
Amélie N. Dreiss*, Charlène A. Ruppli, Fréderic Oberli, Sylvain Antoniazza, Isabelle Henry, \\ Alexandre Roulin \\ Department of Ecology and Evolution, University of Lausanne, Lausanne, Switzerland
}

\section{A R T I C L E I N F O}

\section{Article history:}

Received 4 December 2012

Initial acceptance 25 February 2013

Final acceptance 8 April 2013

Available online 31 May 2013

MS. number: 12-00915

\section{Keywords:}

animal communication

barn owl

overlap

sibling negotiation

signal interference

Tyto alba

\begin{abstract}
Animals communicate with conspecifics to resolve conflicts over how resources are shared. Since signals reflect individuals' resource-holding potential and motivation to compete, it is crucial that opponents efficiently transmit and receive information to adjust investment optimally in competitive interactions. Acoustic communication is particularly flexible as it can be quickly modulated according to background noise and audience. Diverse mechanisms have evolved to minimize acoustic signal interference, one being the avoidance of signal overlap by adjusting the timing of call production to alternate calls with those of competitors. However, the occurrence and function of overlap avoidance in the resolution of competition among relatives have barely been studied. Using young barn owl siblings, Tyto alba, which vocally negotiate over who will have priority access to food provided by parents, we investigated the extent to which nestlings avoid calling simultaneously and the function of this behaviour. We found that nestlings overlapped both their live siblings' calls and experimentally broadcast calls at least five times less often than expected at random. Furthermore, a focal nestling engaged more intensely in vocal negotiation when competing with nestmates that called simultaneously compared to those that did not overlap their respective calls. This suggests that barn owl nestlings avoid calling simultaneously, as overlapped calls are less efficient at deterring siblings from competing. Overlap avoidance reduces signal interference and, as a consequence, would improve the efficiency of communication among kin. ๔ 2013 The Association for the Study of Animal Behaviour. Published by Elsevier Ltd. All rights reserved.
\end{abstract}

Animals are often in conflict over limited resources. To avoid the cost of physical competition, animals communicate with one another to indicate their motivation and competitive ability to contest resources (Parker 1974; Maynard Smith 1982). Provided that communication entails costs, and hence reliably reflects signallers' motivation to compete, the individuals that invest more effort in signalling have priority access to resources. Individuals facing competitors that display high motivation are more likely to give up a contest for which the outcome is predictable (Parker 1974). This phenomenon is reinforced when competitors are kin (Hamilton 1964), since a less motivated individual derives benefits by giving up a contest not only because it avoids competing for an unlikely winning outcome, but also because the contested resources are consumed by a genetically related individual, thus providing inclusive fitness benefits. To advertise their motivation to compete, body condition or social status, conspecifics have not only to produce signals efficiently, but also to perceive the signals of opponents. The avoidance of signal interference is thus an

\footnotetext{
* Correspondence: A. Dreiss, Department of Ecology and Evolution, University of Lausanne, Biophore, 1015 Lausanne, Switzerland.

E-mail address: Amelie.Dreiss@unil.ch (A. N. Dreiss).
}

important component of animal communication (Schwartz 1993; Brumm \& Slabbekoorn 2005).

Animals can communicate using various channels. Acoustic communication is particularly interesting because individuals can modulate vocal signalling rapidly in relation to environmental and social cues (e.g. Remage-Healey \& Bass 2006), and they have the possibility to adopt a large range of signalling strategies (e.g. Todt \& Naguib 2000). As acoustic communication can be obscured by background noise and conspecific interference, various mechanisms have evolved to ensure that signals of different individuals can be discriminated by conspecifics. For instance, the human auditory system has the ability to discriminate between different speakers in a crowd even when the sounds are produced simultaneously, the so-called cocktail party problem (Aubin \& Jouventin 1998; Bee \& Micheyl 2008). Among species of insects, frogs and birds that vocalize in large groups or in noisy environments, individuals shift their call features to avoid overlapping in frequency, so that their vocalizations can be distinguished from one another (Narins \& Zelick 1988; Römer \& Bailey 1998; Slabbekoorn \& Peet 2003). Production of acoustic signals in groups can also be set by temporal organization rules (Ficken et al. 1974; Gerhardt 1994) and alternating acoustic signals, referred to as antiphonal calling, has been documented in groups of bats (Carter et al. 2008) and in 
numerous birds (Stokes \& Williams 1968). Some primates can even detect and wait for silent windows to vocalize (Versace et al. 2008). This temporal organization leads to an alternation of vocal signals, reducing the risk of individuals calling simultaneously.

A particular situation occurs when individuals communicating with one another belong to the same family. In some altricial species, siblings vocalize in the absence of parents to communicate their motivation to compete with one another (Roulin et al. 2000; Johnstone \& Roulin 2003; Bulmer et al. 2008; Magrath et al. 2010). This form of communication, referred to as 'sibling negotiation', reduces the level of sibling competition, and is more likely to evolve in species in which nestmates are full rather than half siblings and when the cost of sibling competition is high rather than low (Johnstone \& Roulin 2003). Such vocal exchanges are usually not heard by parents and therefore cannot be interpreted as a form of begging behaviour that evolved to convey honest information to parents (Roulin et al. 2000). In the barn owl, Tyto alba, the single food item brought by a parent is indivisible and only one offspring is fed per parental feeding visit. Therefore, each nestling should invest more effort in negotiation when its chance of outcompeting its siblings increases (Johnstone \& Roulin 2003). Hence, it is essential that each nestling assesses the level of competitiveness and motivation of all surrounding siblings, to invest effort optimally in sibling competition. Hungrier individuals vocalize at a higher rate with longer calls to signal to nestmates their higher motivation to compete for the next food item to arrive (Roulin 2002; Dreiss et al. 2010b), which would reduce sibling begging towards parents and thus the cost of sibling competition (Johnstone \& Roulin 2003). This intense vocal behaviour of hungry individuals induces siblings to reduce their vocalizations in the absence (sib-sib negotiation) and presence (begging) of parents and hence to withdraw momentarily from the contest over the next food item (Roulin 2002; Dreiss et al. 2010b). Thus, in the absence of parents, nestlings need to hear and be heard, to adjust investment optimally in sibling competition once parents arrive with food. During a single night nestlings can produce thousands of calls, implying that this communication system may be costly in terms of energy and time invested (e.g. in another system: Kilner 2001; RodriguezGironés et al. 2001; Chappell \& Bachman 2002). We hypothesized that, to maximize transmission of vocal signals between siblings, nestlings avoid calling simultaneously, otherwise they may have to call even more often to transfer the same amount of information. Although a previous study in the European starling, Sturnus vulgaris, observed that siblings would avoid overlapping their vocalizations when parents are away (Chaiken 1990), no experimental test has yet been performed.

We tested this hypothesis of overlap avoidance in nestling barn owls experimentally, by studying unmanipulated vocal interactions between pairs of nestlings (i.e. dyads). Siblings differ in age owing to a pronounced hatching asynchrony, which results in asymmetries in the competitiveness of nestmates and different vocal behaviours (Roulin 2004). Given their stronger competitive abilities, seniors can usually outcompete their siblings while investing relatively less in vocalizations and they are less sensitive to the vocal behaviour of their junior siblings (Roulin 2004). We thus recorded naturally occurring vocal interactions between dyads of siblings that were either both food deprived or both food satiated, each dyad comprising one senior and one junior nestling. If overlap is an aggressive or competitive signal itself, it should be used more often by hungry individuals and more competitive individuals (here seniors). Alternatively, if nestlings avoid calling simultaneously to transfer information efficiently, they should avoid overlap whatever the competitive situation. We tested whether the degree to which an individual avoids calling simultaneously to its sibling varies with motivation, that is, its level of hunger, and with competitiveness, that is, between juniors and seniors, and with developmental stage (i.e. absolute age).

In such a design, each nestling produces calls at variable rhythms and hence the observation of siblings not calling simultaneously may simply result from the fact that siblings have different timedependent activity patterns. Hence, to tackle the hypothesis that barn owl siblings actively avoid calling simultaneously, we analysed nestling response to playback experiments with variable call rate. In natural vocal interactions, the rhythm of nestlings' calls varies from loose clusters of rapid calls to relatively regular (see for instance Roulin et al. 2009). We thus analysed the vocal response of singleton nestlings (nestlings placed individually in an experimental box) to two different playback soundtracks, one with negotiation calls broadcast at random (unpredictable) time points, and another broadcast at a constant (predictable) rhythm. If barn owl siblings minimize acoustic signal interference, we predicted that individuals would call simultaneously to their siblings or the playback less often than expected at random, regardless of the rhythm at which calls are produced. To test the effect of competition on nestling propensity to avoid call overlap, we also varied playback call rate, call duration and number of calls. If overlap is a competitive signal, it should occur more often in competitive situations, when call rate and call duration are high (Roulin et al. 2009) and potentially when more individuals are calling. Conversely, nestlings are expected to avoid calling simultaneously in all situations if overlap avoidance efficiently allows information transfer.

Assuming that the adaptive function of not overlapping calls is to improve the efficiency of communication, we predicted that an individual will more efficiently deter its siblings from competing if it does not call simultaneously to them. To test this prediction we performed a third experiment. Singleton nestlings were broadcast pairs of calls produced by two individuals that were separated by a short pause (no overlap referred to as '0\% overlap' treatment), overlapped on half of their duration ('50\% overlap' treatment) or entirely ('100\% overlap' treatment; Fig. 1). While broadcasting these

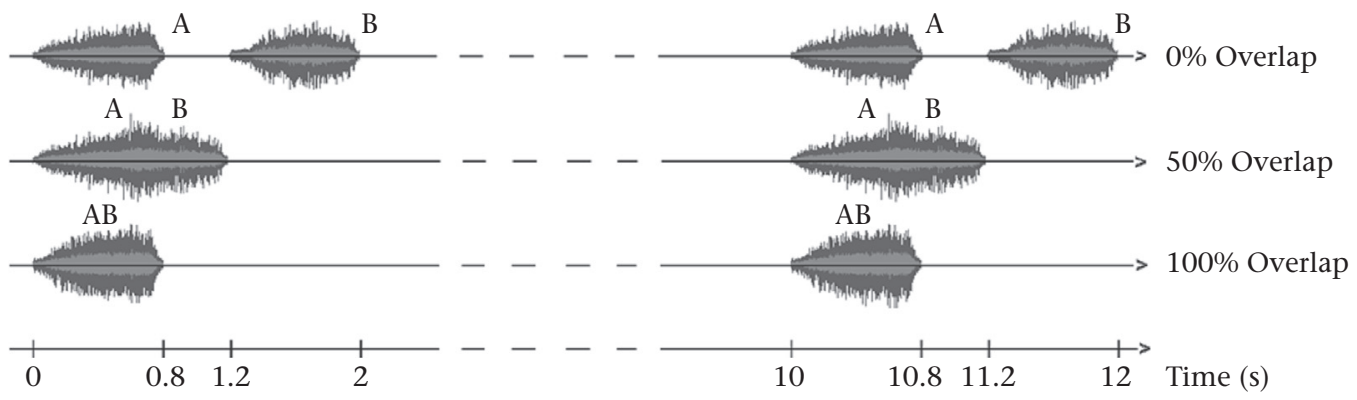

Figure 1. Diagram of the three playback treatments (experiment 3). Each treatment consisted of the repetition of a pair of calls from two different unfamiliar nestlings (A and B) every $10 \mathrm{~s}$. ' $0 \%$ overlap': calls of A and B are separated by a pause of $0.4 \mathrm{~s}$; ‘ $50 \%$ overlap': calls of A and B overlapped on $50 \%$ of their duration; and ' $100 \%$ overlap': calls of A and B are each overlapped on $100 \%$ of their duration. Treatments were randomly ordered across singleton nestlings. 
calls we simultaneously recorded the vocal response of the focal singleton nestlings to determine whether they adjusted their vocalization behaviour in relation to the degree of overlap between the calls of the two broadcast individuals. Based on the sibling negotiation hypothesis proposing that an individual retreats from a food contest when facing competitors that are hungrier and produce more and longer calls (Roulin 2002; Dreiss et al. 2010b), we predicted that singleton nestlings would vocalize more intensely when listening to overlapped than nonoverlapped calls, since the overall duration of the signals produced is lower when nestmates vocalize simultaneously.

\section{METHODS}

\section{General Procedures}

The study was performed in western Switzerland $\left(46^{\circ} 4^{\prime} \mathrm{N}, 6^{\circ} 5^{\prime} \mathrm{E}\right)$ on a population of wild barn owls breeding in nestboxes located in barns. When nestlings were 18-45 days old (mean $\pm \mathrm{SE}=34.5 \pm 0.3$ days), we brought them to the University of Lausanne. Our previous studies showed that in captivity they behave vocally as in nature (Roulin et al. 2009) and are not physiologically stressed (Dreiss et al. 2010a). Among the three to nine nestlings of a brood, we always left at least one nestling in the natural nest to make sure parents did not abandon their brood. At that age, owlets are thermoindependent and able to eat prey items without maternal help. For these reasons, the mother does not sleep with her offspring during the daylight hours and comes back only at night to deliver food items. Thus, we carried out our laboratory experiments on offspring that were used to the mother's absence.

\section{Experiment 1: Overlap Avoidance Between Sibling Pairs}

In 2008, we recorded 78 dyads of siblings (21-45 days old, 83 males, 71 females, two individuals of unknown sex) from 41 different broods. For 25 broods that included four nestlings, we matched two dyads of siblings, and for six broods that included six nestlings we matched three dyads of siblings. Siblings were kept for 3 nights in an experimental nestbox similar to the one in which they were reared in natural conditions $(62 \times 56 \mathrm{~cm}$ and $37 \mathrm{~cm}$ high), except that the box was divided in half by a thin wooden wall pierced with five holes at the top. We placed one nestling on each side of the wall, randomly chosen, so that siblings could hear each other without interacting visually or physically. Each dyad of nestlings comprised a senior individual and a junior sibling, which was on average 5 days younger (range 1-15 days). After a first night of acclimation, each dyad of siblings was recorded over 2 nights, from 1930 to 2330 hours. One night we deprived the nestling dyad of food (no food given during the preceding $28 \mathrm{~h}$ ) and the other night we gave them enough to satiate them (from 0000 to 1600 hours on the recording day we offered $130 \mathrm{~g}$ (three to four) of laboratory mice, which exceeds their daily food requirement of about $67 \mathrm{~g}$;
Durant \& Handrich 1998), with the order of the two treatments being randomly assigned in time across dyads. Individuals that were deprived of food on the first night were randomly chosen, since their mean body mass at the start of the experiment was similar to the mean body mass of individuals receiving the ad libitum treatment the first night (Student's $t$ test: $t_{202}=0.63$, $P=0.53$ ).

As in natural conditions (A. N. Dreiss, personal observation), it sometimes happened that one of the two individuals did not vocalize during the $4.5 \mathrm{~h}$ recording period. Among the 78 dyads of siblings this occurred in 10 dyads (13\%) when food deprived and in 20 dyads (26\%) when satiated. Since our goal was to study vocal interactions between two individuals, we excluded these dyads of siblings to perform statistical analyses (Table 1 ).

\section{Experiment 2: Overlap Avoidance of Playback Calls}

\section{Experimental design}

In 2009 and 2010, we brought free-living nestlings to the University of Lausanne for the playback experiments, in the same rooms as those in which we recorded dyadic vocal interactions. Nestlings were placed alone in one side of the same experimental nestboxes, with a loudspeaker (near05 experience, ESI Audiotechnik GmbH, Leonberg, Germany) in the other side to broadcast a prerecorded playback sequence.

In 2009, we broadcast prerecorded calls at unpredictable time points (hereafter denoted 'unpredictable playback') and at varying call rate and duration to 54 nestlings (27-44-day-old nestlings, 20 males, 33 females, one individual of unknown sex) from 16 different broods. We broadcast the calls at three different rates $(2,6$ and 10 calls $/ \mathrm{min}$ ) and three different durations (0.6, 0.8 and $1 \mathrm{~s}$ ). These values of call duration and call rate correspond to the mean values and to the lowest and highest $10 \%$ of the distributions observed in the two-chick broods. These two acoustic variables are important parameters used in sibling negotiation (Roulin et al. 2009; Dreiss et al. 2010b). We built nine playback sequences lasting $15 \mathrm{~min}$ each, which were separated from one another by $10 \mathrm{~min}$ of silence. These sequences correspond to the combination of the three different call rates, with the three different call durations. We inserted calls randomly and thus unpredictably in the soundtracks, except that two successive calls were separated by at least $1 \mathrm{~s}$. A pause of $1 \mathrm{~s}$ or less between two successive calls produced by the same individual was observed in only $0.03 \%$ of the cases (mean $\pm S D=15.3 \pm 106.4 \mathrm{~s}$, range $0.7-7259.2 \mathrm{~s}$ ).

In 2010, we broadcast calls at a constant rhythm (hereafter denoted 'predictable playback') with a varying call rate and number of playback individuals to 96 singleton nestlings (18-41 days old, 47 males, 48 females, one individual of unknown sex) from 22 broods. We broadcast sequences of $20 \mathrm{~min}$ at three different rates (2, 6 and 10 calls/min), each sequence containing the calls of one, two or five different playback individuals, with the calls of different individuals being allocated randomly in the playback sequence

Table 1

Summary of the laboratory experiments used to assess the occurrence of call overlap in nestling barn owls

\begin{tabular}{|c|c|c|c|c|c|c|c|}
\hline \multicolumn{2}{|c|}{ Experiment } & \multirow{2}{*}{$\begin{array}{l}\text { Year } \\
2008\end{array}$} & \multirow{2}{*}{$\begin{array}{l}\text { Total number of } \\
\text { nestlings }\end{array}$} & \multirow{2}{*}{$\begin{array}{l}\begin{array}{l}\text { Sample size } \\
\text { analysed }\end{array} \\
136 \text { (68 dyads) }\end{array}$} & \multirow{2}{*}{$\begin{array}{l}\text { Number of } \\
\text { broods }\end{array}$} & \multirow{2}{*}{$\begin{array}{l}\text { Time of } \\
\text { recording }\end{array}$} & \multirow{2}{*}{$\begin{array}{l}\text { Number of calls recordec } \\
\text { per nestling (mean } \pm \text { SE) }\end{array}$} \\
\hline 1 & $\begin{array}{l}\text { Dyadic vocal interaction between } \\
\text { food-deprived siblings }\end{array}$ & & & & & & \\
\hline & $\begin{array}{l}\text { Dyadic vocal interaction between } \\
\text { food-satiated siblings }\end{array}$ & 2008 & 156 (78 dyads) & 116 (58 dyads) & 41 & $1930-2330$ & $895 \pm 88$ \\
\hline \multirow[t]{2}{*}{2} & Unpredictable playback & 2009 & 54 & 48 & 16 & $2100-0045$ & $59 \pm 3$ \\
\hline & Predictable playback & 2010 & 96 & 65 & 22 & $2100-0130$ & $62 \pm 3$ \\
\hline 3 & Response to overlapping calls & 2010 & 70 & 57 & 16 & $0150-0320$ & $216 \pm 24$ \\
\hline
\end{tabular}

The sample size analysed was the number of dyads in which both siblings vocalized or the number of singleton nestlings that vocalized in response to playback. 
(in each sequence all playback individuals produced the same number of calls). By broadcasting the calls from one to five playback individuals, we tried to mimic the several siblings naturally present in the nest (up to nine in our study population). This experiment thus consisted of nine consecutive sequences of $20 \mathrm{~min}$ each, separated by $10 \mathrm{~min}$ of silence, corresponding to the combination of three different call rates by three different numbers of playback individuals. Calls were inserted in a random order, but at regular time intervals; every 30,20 or $6 \mathrm{~s}$ to obtain playback sequences that differed in call rate.

In both the unpredictable and predictable playback experiments, the focal nestlings that listened to the playback were not fed between 0800 hours the preceding morning until the start of the experiment at 2100 hours as is usual in natural conditions (A. N. Dreiss, personal observation). The experiments were conducted on the third night that nestlings spent in the laboratory for the unpredictable playback (2009) and on the second night for the predictable playback (2010). In 2009, six of the 54 nestlings (11\%) did not call during the recording session taking place between 2100 and 0045 hours. In 2010, 31 nestlings (32\%) did not vocalize during the recording session taking place from 2100 to 0130 hours (Table 1) (comparison of the frequency of noncallers across years: $\left.\chi^{2}=34.5, P<0.0001\right)$

\section{Construction of Playback Soundtracks}

We extracted the calls used to build all playback soundtracks from the dialogues of sibling dyads recorded in 2008. Calls were selected for their duration and not modified, except for loudness, which was standardized using Audacity v.1.3 Beta freeware (http:// audacity.sourceforge.net). This manipulation did not affect other acoustic parameters. We had to use this procedure because the recorded individuals were not always positioned at a constant distance from the microphone, as they moved freely in the box. We built all playback sequences using an automatic program in Matlab v.7.7 (MathWorks, Natick, MA, U.S.A.) that inserted the calls at random in the experiment performed in 2009 (unpredictable playback) or at constant intervals in the experiment performed in 2010 (predictable playback).

To build the unpredictable playback soundtracks for 2009, we selected calls from 16 nestlings, with 30 calls each (six males, 10 females; aged 28-45 days), corresponding to 10 calls of three different categories of call duration. Across the nine sequences played back to each singleton nestling, we used the calls from two of the 16 individuals, which we chose randomly, with one sequence of 15 min always containing the calls of a single playback individual. To avoid pseudoreplication (Kroodsma et al. 2001), the playback soundtracks broadcast were unique, as call order and timing as well as the order of the nine sequences were randomized for each singleton nestling.

To build the predictable playback soundtracks for 2010, we used the 10 selected calls of $0.6 \mathrm{~s}$ from the 16 playback nestlings broadcast in 2009. We built a unique playback soundtrack for each nestling by randomizing the order of the nine sequences and the order of the calls of playback individuals in each sequence.

\section{Experiment 3: Vocal Response to Overlapping Calls}

\section{Experimental design}

In 2010, we performed a second playback experiment 20 min after the 'predictable playback' starting at 0150 hours to examine the potential adaptive function of not overlapping calls. For logistical reasons, this was done with 70 of the 96 nestlings. We broadcast three playback treatments in a row, each sequence lasting $20 \mathrm{~min}$, with periods of $10 \mathrm{~min}$ of silence between two sequences. In the three treatments, we broadcast pairs of calls from two individuals with one individual always calling before the other. Pairs of calls were regularly spaced across the 20 min treatments, starting every $10 \mathrm{~s}$ (Fig. 1). In the first treatment the pairs of calls did not overlap, that is, an individual always produced its calls $0.4 \mathrm{~s}$ after the call of the other individual ended; this treatment is referred to as '0\% overlap' (Fig. 1 ). In the second treatment, denoted ' $50 \%$ overlap' (Fig. 1), an individual always produced its calls $0.4 \mathrm{~s}$ after the call of the other individual started. Therefore, the calls of the two individuals overlapped for half of their time (i.e. during $0.4 \mathrm{~s}$ ). In the third treatment, denoted ' $100 \%$ overlap' (Fig. 1), the calls of the two individuals were produced simultaneously. We randomized the order of the three treatments across nestlings. Of the 70 nestlings, 13 (19\%) did not call in all three playback treatments and were excluded from analysis.

\section{Construction of playback soundtracks}

To build the playback soundtracks, we used the calls of 12 individuals recorded in 2008, which were different from those used in the preceding 'predictable playback' experiment. We randomly selected 10 calls per playback individual that all lasted $0.8 \mathrm{~s}$, which corresponds to the mean call duration that food-deprived individuals produce in natural dialogues (Dreiss et al. 2013) and that were also standardized for loudness using Audacity v.1.3. We broadcast pairs of calls and in each 20 min long soundtrack we always broadcast a given playback individual before the other; in 27 of 57 cases the individual that was first broadcast in each pair of calls was younger than the other individual, in four cases the two individuals had the same age and in the other 26 cases it was older. In 35 of the playback soundtracks, the two individuals were male and female, in eight soundtracks they were males and in 14 soundtracks they were females. In the playback soundtracks we always broadcast one individual after the other, and each of the 12 playback individuals was as often broadcast first as second $\left(\chi_{11}^{2}=3.0, P=0.99\right)$.

Each playback lasted $20 \mathrm{~min}$ and comprised 120 pairs of calls, corresponding to 10 distinct pairs of calls repeated 12 times in a random order. A maximum of two playbacks were built with the same individuals, but the order of the calls of the two playback individuals was reversed. Each singleton nestling thus heard a unique playback.

\section{Acoustic recordings}

We considered that nestlings overlapped a call of their counterpart (their sibling or the playback) when they initiated their calls before the call of their counterpart ended. Negotiation calls are a simple hiss, continuous (containing no silent pause within) and quasistationary (i.e. the frequencies of one call are more or less constant from its beginning to the end; see Figure 10 in Bühler \& Epple 1980).

We simultaneously recorded vocal interactions of dyads of siblings using two microphones (MC930, Beyerdynamic GmbH \& Co KG, Heilbronn, Germany) oriented in opposite directions, each facing one bird and connected to a multichannel recording system managed by Cubase v.5.2 software (Steinberg Media Technologies $\mathrm{GmbH}$, Hamburg, Germany). The two recording soundtracks were analysed with Matlab v.7.7 (MathWorks, Natick, MA, U.S.A.) to assign calls to each individual of a dyad and to measure precise timing of call onset and call duration (see script in Dreiss et al. 2013).

We recorded the vocal response of singleton nestlings responding to playback using a single microphone facing the nestling. We again used Matlab v.7.7 to assign calls to the singleton nestling and the playback and to measure the timing of call onset as well as the duration of each call (see script in Dreiss et al. 2013). 


\section{Statistical Analyses}

\section{Experiment 1: overlap avoidance between sibling pairs}

We used a randomization procedure to investigate whether nestlings' overlapping of each other's calls was different from calling at random time points. For each vocal exchange between dyads of siblings, we randomized the calls produced by both siblings with respect to time of call onset. Duration of the vocal exchange and the number and duration of the calls were thus kept unchanged. Since barn owl nestlings can naturally produce two successive calls with barely any pause between them (we recorded five pauses of 0 to $0.1 \mathrm{~s}$ among 10000 recorded pauses), we did not constrain this randomization with respect to pause duration between two calls of the same individual. The randomization of the calls of each sibling of a dyad was repeated 1000 times to generate a null distribution of call overlaps for each dyad of siblings. This null distribution was then compared with the observed call overlap in the dyad of siblings to calculate a $P$ value. Global probability of overlap across all dyads of siblings was determined with a Fisher combined probability test. We analysed juniors and seniors separately because they are not statistically independent. We corrected for multiple testing with Bonferroni correction (see Results). We also performed distinct analyses for dyads of food-deprived and food-satiated siblings, as each nestling was recorded in both states.

\section{Experiment 2: overlap avoidance of playback calls}

We applied a similar randomization procedure as described above to assess whether nestlings avoid overlapping the broadcast calls. For each playback sequence, we randomized the calls produced by the focal nestling with respect to time of onset 1000 times and we compared the obtained null distribution of call overlap with the playback calls to the observed call overlap in the experiment. To analyse independent data in Fisher combined probability tests, so that each nestling appeared only once per test, we performed separate analyses for each of the nine combinations of playback treatment (call rate and call duration for unpredictable playback; call rate and number of individuals for predictable playback).

\section{Experiment 3: vocal response to overlapping calls}

We analysed the number and duration of calls produced by the focal individual that was listening to the playback during each playback treatment using, respectively, a generalized mixed model with a Poisson distribution and a log link and a linear mixed model. We included the independent term 'age of the focal nestling' and the cofactors 'playback treatment' and 'order in which each treatment was broadcast' (first, second or third). To control for the repeated measurements per brood and nestling, we included nestling identity nested in the identity of the brood from which individuals originated as random factors. We also applied the same randomization procedure as described for experiment 2 to assess whether nestlings avoided overlapping differently depending on the degree of overlap between broadcast individuals.

\section{Experiments 1-3: influence of other factors}

To analyse the propensity of vocal overlapping, we ran generalized mixed models with a binomial error distribution and a logit link, with, as dependent term, the number of calls produced by the focal nestling that overlapped a call of its counterpart or the playback, divided by the total number of calls produced by the focal nestling, using SAS v.9.1 (SAS Institute Inc., Cary, NC, U.S.A.). For experiment 1 , we included the independent terms age and number of calls produced by the sibling and the cofactors food treatment and seniority, that is, whether the nestling was the junior (younger) or the senior (older) of the dyad. For experiment 2 , we included the independent terms age, playback order (1-9), call rate and call duration for unpredictable playback, and call rate and number of playback individuals for predictable playback. For experiment 3 , we included the independent terms age, playback order (1-3) and playback treatment $(0,50$ or $100 \%$ overlap). To control for the repeated measurements, we included nestling identity nested in the broods from which individuals originated as random factors. For experiment 1 we also included the identity of the sibling dyad as a random factor.

Means are provided \pm SE.

\section{Ethical Note}

Nestlings were put back into their nest at the end of the study period (3 nights in 2008 and 2009, 2 nights in 2010). Removing several nestlings from a nest for 2 or 3 nights never induced parents to abandon their brood. We always left one or two nestlings in the natural nest and we had already observed that parents do not adjust feeding rate to short-term variations in food need (Roulin et al. 2000). Nestlings were transported in opaque aerated plastic boxes, with a foam floor. In the laboratory, nestlings were not physiologically stressed, as shown by the absence of a rise in baseline corticosterone level compared to the situation prevailing under natural, undisturbed conditions (Dreiss et al. 2010a) and we did not observe behavioural signs of stress, as nestlings ate a normal amount of food when offered, did not show stereotypical movements and did not produce antipredator hissing sounds. Experimental boxes were connected to the outside with a pipe $10 \mathrm{~cm}$ in diameter and $20 \mathrm{~cm}$ long, allowing air and natural light to enter. Blood samples (around $20 \mu \mathrm{l}$ ) for corticosterone assay were taken by puncturing the brachial vein and collecting the blood with heparinized capillary tubes (see methods and results details in Dreiss et al. 2010a). We fed nestlings with laboratory mice, Mus musculus, euthanized by $\mathrm{CO}_{2}$, bought frozen from an animal house (Reptiles Farm, Servion, Switzerland). Barn owl nestlings can fast naturally for 1-2 rainy nights (A. N. Dreiss, personal observations). Keeping owlets at the university did not negatively affect their body condition since mean body mass at fledgling and survival up to fledgling did not differ significantly between nestlings brought to the university and nestlings left in their nest (body mass at fledgling stage: recorded: $358 \pm 2 \mathrm{~g}$; nonrecorded: $353 \pm 3 \mathrm{~g}$; Wilcoxon test: $Z=1.6, N=389, P=0.11$; mortality: recorded: $10 \%$; nonrecorded: 16\%: Wilcoxon test: $Z=1.6, N=437, P=0.10$ ). Nestlings were captured by hand in their nestbox and marked with aluminium rings. The experiments were approved by the veterinary services of Canton de Vaud (Form No 2109.1).

\section{RESULTS}

\section{Experiment 1: Overlap Avoidance Between Sibling Pairs}

Food-deprived and food-satiated nestlings overlapped the calls of their siblings (which were in a similar food state) in only $1.04 \pm 0.13 \%$ and $0.81 \pm 0.04 \%$ of cases, respectively, which is 7.3 and 4.6 times less often than expected at random (Fig. 2; Fisher combined probability tests: $\chi^{2}=908$ and 897 for junior and senior food-deprived siblings, respectively; $\chi^{2}=553$ and 594 for junior and senior food-satiated siblings; all $P<0.0001$ ). Food treatment, absolute age and seniority (junior or senior) did not have any significant effect on the proportion of observed overlapped calls (generalized mixed model: food treatment: $F_{1,95}=1.30, P=0.26$; absolute age: $F_{1,95}=0.26, P=0.61$; seniority: $F_{1,95}=0.70, P=0.40$; number of calls of partner sibling: $F_{1,95}=116.01, P<0.0001$ ). 


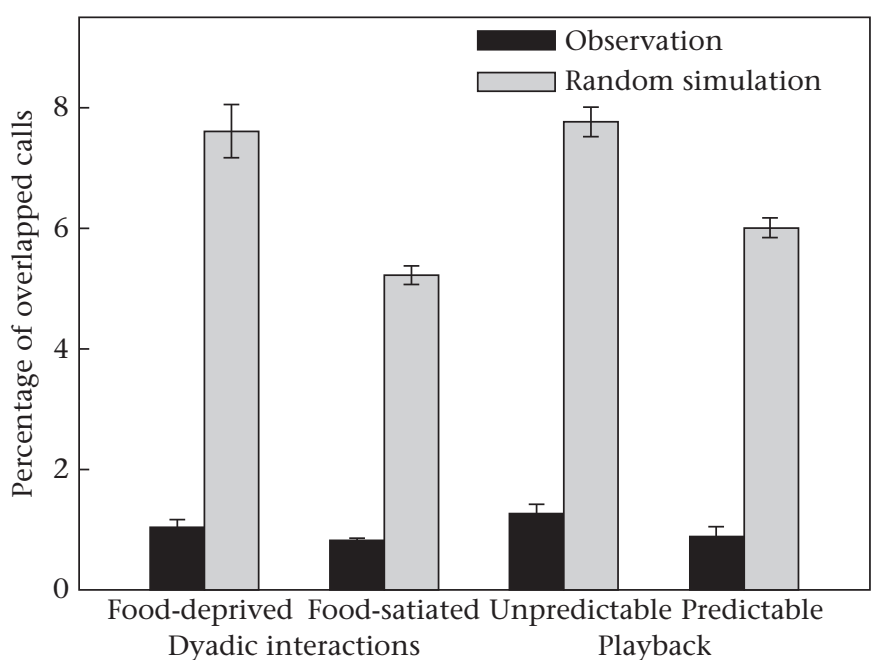

Figure 2. Observed (black) and expected (grey) mean percentage of overlapped calls \pm SE of barn owl nestlings (experiments 1and 2). By definition, a nestling produced 'overlapped calls' when it starts a call before a call of its sibling or before a broadcast call has ended. Nestlings either exchanged vocalizations with a sibling during unmanipulated vocal dyadic interactions, in a food-deprived or food-satiated state, or responded to prerecorded playback sequences containing calls inserted at an unpredictable random timing or at a predictable fixed timing.

\section{Experiment 2: Overlap Avoidance of Playback Calls}

The focal singleton nestlings vocalized simultaneously to playback calls only $1.27 \pm 0.15 \%$ and $0.88 \pm 0.16 \%$ of the time for unpredictable and predictable playbacks, respectively, which is 4.7 and 8.8 times less often than expected at random (Fig. 2). Nestlings overlapped unpredictable playback significantly less than expected by chance in all playback sequences, except for the sequence that comprised 2 calls/min and calls of $0.6 \mathrm{~s}$ (Table 2). Predictable playback was overlapped less than expected by chance in six of nine sequences (Table 2; for the three sequences that comprised 2 calls $/ \mathrm{min}$, the test was not significant after Bonferroni correction). At random, an average of $2.4 \pm 0.3 \%$ of calls overlapped the playback during the sequences containing 2 calls/min. This very low proportion explains why the difference between observed overlap and random overlap was less pronounced during playback of 2 calls/min. A higher proportion of nestlings' calls overlapped playback calls when the playback call rate was higher (generalized mixed model with nestling identity nested in the brood as random factors: unpredictable playback: $0.13 \pm 0.02, F_{1,233}=38.69, P<0.0001$; predictable playback: $\left.0.20 \pm 0.03, F_{1,204}=61.24, P<0.0001\right)$. Age of the singleton nestling, playback order and playback treatment (call duration and number of playback individuals) did not have any effect on the proportion of observed overlapped calls (generalized mixed model: unpredictable playback: nestling age: $F_{1,231}=0.01, P=0.93$; call duration: $F_{1,231}=1.16, \quad P=0.32 ;$ playback order: $F_{1,205}=2.84, \quad P=0.09$; predictable playback: nestling age: $F_{1,201}=0.95, P=0.33$; number of broadcast individuals: $F_{1,201}=0.13, P=0.88$; playback order: $\left.F_{1,233}=2.13, P=0.15\right)$.

\section{Experiment 3: Vocal Response to Overlapping Calls}

Singleton nestlings produced more and longer calls when the degree of overlap between the broadcast calls was higher (Fig. 3, Table 3). Nestlings produced shorter calls when they were listening to playback calls that did not overlap (i.e. '0\% overlap' treatment) compared to calls that overlapped for only half of their duration (i.e. '50\% overlap' treatment) or overlapped entirely (i.e. '100\% overlaps' treatment; estimates \pm SE: 0 versus $50 \%:-0.06 \pm 0.02 ; t_{106}=-3.4$, $P=0.001 ; 50$ versus $\left.100 \%:-0.05 \pm 0.02 ; t_{106}=-2.7, P=0.008\right)$. They also produced fewer calls when hearing calls that overlapped during half of their duration (i.e. '50\% overlap' treatment) than for $100 \%$ of their duration (i.e. '100\% overlaps' treatment; estimates \pm SE: 0 versus $50 \%$ : $-0.02 \pm 0.02 ; t_{114}=-0.98, P=0.32$; 50 versus $100 \%$ : $\left.-0.12 \pm 0.02 ; t_{114}=-5.34, P<0.001\right)$. Number of calls produced decreased with playback order and with nestling age (Table 3; estimates \pm SE: first versus second playback treatment: $0.22 \pm 0.02 ; t_{114}=9.93, P<0.001$; second versus third: $0.05 \pm 0.02 ; t_{114}=2.14, P=0.034 ;$ age: $\left.-0.09 \pm 0.04\right)$.

As expected, nestlings avoided calling simultaneously with playback calls (Fisher combined probability tests: '0\% overlap': $\chi^{2}=397, P<0.0001 ;$ ' $50 \%$ overlap': $\chi^{2}=297, P<0.0001 ;$ ' $100 \%$ overlap': $\left.\chi^{2}=407, P<0.0001\right)$. The proportion of nestlings' calls overlapping playback was not related to playback treatment $\left(F_{2,145}=0.95, P=0.39\right)$, test playback order $\left(F_{1,145}=1.03, P=0.31\right)$ or nestling age $\left(F_{1,145}=0.35, P=0.55\right)$.

\section{DISCUSSION}

We have shown that nestling barn owls, which are known to negotiate vocally with one another for food resources delivered by parents (Roulin et al. 2000; Roulin 2002), have developed a mechanism to avoid calling simultaneously. Using both correlative and playback approaches, we found that nestlings preferentially called one after the other rather than simultaneously. This phenomenon of overlap avoidance suggests that accuracy of signal transmission is essential in this sibling communication system. This statement is consistent with the result showing that nestlings negotiated vocally at a higher level when playback calls overlapped than when they did not overlap.

Our results show that nestlings do not vary in the extent of overlap avoidance according to their dominance status (i.e. absolute age and seniority) and hunger level, which reflects motivation to compete for food. This absence of variation across individuals and food states suggests that overlap is not used as a competitive signal itself, to deter competitors from calling, contrary to some observations in songbirds (Todt \& Naguib 2000). Antiphonal patterns enable interacting individuals to avoid signal interference and

Table 2

Result of Fisher combined probability tests for the different sequences of playback

\begin{tabular}{|c|c|c|c|c|c|c|c|c|}
\hline & & & \multicolumn{3}{|c|}{ Playback call duration (s) } & \multicolumn{3}{|c|}{ Number of playback individuals } \\
\hline & & & 0.6 & 0.8 & 1 & 1 & 2 & 5 \\
\hline \multirow{6}{*}{$\begin{array}{l}\text { Playback call rate } \\
\text { (calls/min) }\end{array}$} & 2 & $\chi^{2}$ & 104.8 & 142.4 & 165.7 & 125.8 & 111.9 & 119.8 \\
\hline & & $P$ & 0.062 & 0.0001 & $<0.0001$ & 0.005 & 0.044 & 0.02 \\
\hline & 6 & $\chi^{2}$ & 212.3 & 229.0 & 266.0 & 199.0 & 237.8 & 222.5 \\
\hline & & $P$ & $<0.0001$ & $<0.0001$ & $<0.0001$ & $<0.0001$ & $<0.0001$ & $<0.0001$ \\
\hline & 10 & $\chi^{2}$ & 304.6 & 294.8 & 230.1 & 256.7 & 255.9 & 303.1 \\
\hline & & $\stackrel{n}{P}$ & $<0.0001$ & $<0.0001$ & $<0.0001$ & $<0.0001$ & $<0.0001$ & $<0.0001$ \\
\hline
\end{tabular}

$P$ values below the 0.005 threshold (after Bonferroni correction) indicate that nestlings overlapped playback calls significantly less than expected by chance. 

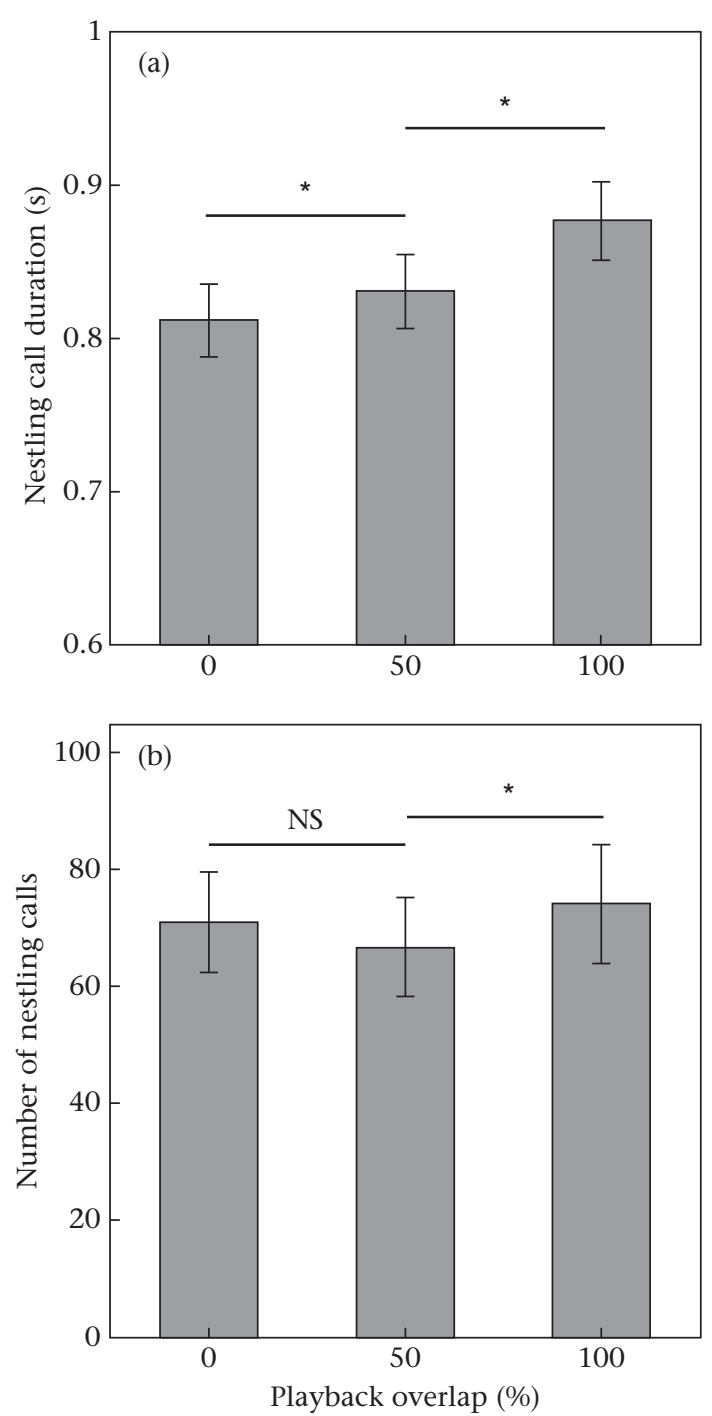

Figure 3. (a) Nestling mean call duration $\pm \mathrm{SE}$ and (b) mean number of calls $\pm \mathrm{SE}$ in response to pairs of playback calls that overlapped at 0,50 or $100 \%$ (experiment 3 ). ${ }^{*} P<0.05$.

thus the jamming of information contained in signals. In some communication systems, the end of calls is more variable and thus carries more information than the beginning (Todt \& Naguib 2000). An individual that overlaps a counterpart's vocal signal may benefit from masking the competing signal, without losing much of its own signal information (Hultsch \& Todt 1982). This is not the case in the barn owl in which calls are simple hissing sounds implying that the

Table 3

Result of statistical analyses on singleton nestlings' vocal response to pairs of playback calls varying in degree of overlap

\begin{tabular}{|c|c|c|c|c|c|c|}
\hline & \multicolumn{3}{|c|}{ Nestling's call number } & \multicolumn{3}{|c|}{ Nestling's call duration } \\
\hline & $F$ & $d f$ & $P$ & $F$ & $d f$ & $P$ \\
\hline $\begin{array}{l}\text { Test playback order } \\
\text { (1st, 2nd or 3rd) }\end{array}$ & 55.75 & 2,114 & $<0.001$ & 0.22 & 2,104 & 0.80 \\
\hline $\begin{array}{l}\text { Playback treatment } \\
\quad(0,50 \text { or } 100 \% \text { overlap })\end{array}$ & 15.79 & 2,114 & $<0.001$ & 6.47 & 2,106 & 0.002 \\
\hline Nestling age & 7.13 & 2,114 & 0.009 & 0.08 & 1,104 & 0.78 \\
\hline
\end{tabular}

Playback treatments correspond to sequences of playback calls overlapped at 0,50 or $100 \%$ (experiment 3; Fig. 1). Nestling identity nested in the identity of the brood from which individuals originated was set as a random factor. same information is conveyed at the beginning as at the end of calls (Bühler \& Epple 1980). Overlapping birds that start calling before their counterpart has terminated its call would thus suffer the same signal masking as their overlapped counterpart, and thus similarly hinder information transfer. This interpretation is consistent with the fact that a higher degree of overlap between broadcast calls elicited a stronger vocal response from singleton nestlings (Fig. 3). Following the sibling negotiation hypothesis, an individual refrains from competing when its siblings are highly compared to weakly motivated to compete (Roulin 2002). Accordingly, we have repeatedly demonstrated that nestlings retreat from a contest when facing highly vocal siblings (Dreiss et al. 2010b). Hence, overlapped calls less efficiently deter siblings from entering into vocal negotiation, one explanation being that nestlings are less able to perceive their rival's motivation to compete. Alternatively, nestlings that produced calls simultaneously with other siblings (either by overlapping or being overlapped) may be perceived as weaker rivals, possibly because they are unable to produce calls at an appropriate timing. Therefore, calling simultaneously with siblings is counterproductive to negotiating resources.

Call overlap may modify several call features that are important in sibling vocal competition. Call duration and call rate are two of them, and they are known to influence negotiation among barn owl siblings (Roulin et al. 2009; Dreiss et al. 2010b). Call overlap in experiment 3 may have induced a decrease in perceived number of calls if silence is necessary to detect distinct calls and a decrease in perceived signal duration (note that $50 \%$ overlapped calls are longer than each of two nonoverlapped calls, but were less effective at deterring nestlings from competing). Additionally, nestlings may perceive fewer competitor siblings when calls are overlapped, for instance detecting only one nestling calling when two calls are superimposed. Finally, the distribution of sound frequencies (in $\mathrm{Hz}$ ) is affected by call overlap, potentially leading nestlings not to recognize overlapped calls as negotiation signals.

In the sibling negotiation process, although individuals are in conflict over the same resource, two elements promote the evolution of overlap avoidance: the importance of signal exchange for food sharing and the relatedness between competitors (Johnstone \& Roulin 2003). Relatedness affects the intensity of sibling competition, as suggested for instance during begging in nestling barn swallows, Hirundo rustica, which moderate their begging when facing related nestmates (Boncoraglio et al. 2009). During sibling negotiation, genetically related siblings should assess each other's motivation in order to invest optimally in sibling competition. As shown here, vocal overlap seems to blur the signal, which diminishes the efficiency of this negotiation system. Because sibling negotiation is more likely to evolve when the degree of relatedness between nestmates is higher (Johnstone \& Roulin 2003), we conclude that kin selection should promote the evolution of mechanisms that allow nestmates to avoid overlapping their calls. In the barn owl, in which offspring mainly compete with full siblings (Roulin et al. 2004), nestlings may refrain from calling simultaneously to allow their siblings the opportunity to call and/or favour their own signal efficiency. Indeed, sibling negotiation can be considered as a form of altruism between related individuals, since its primary function is to reduce the level of sibling competition.

Sibling competition is particularly prevalent in offspring that still depend on their parents for food resources (Wright \& Leonard 2002) and they often share the same acoustic space to signal their motivation to compete. Offspring must efficiently signal their need towards their parents, which allocate food among their progeny based on these signals (Godfray 1995). When obtaining food mainly depends upon offspring signalling towards their parents and feeding visits are very short, siblings can often not avoid calling simultaneously in an attempt to attract the attention of their parents 
bringing food. Although offspring adjust their level of signalling to that of their siblings (Smith \& Montgomerie 1991; Leonard \& Horn 2001; Blanc et al. 2010), this usually induces individuals to escalate their begging behaviour in an attempt to be louder than their siblings. These adjustments can be interpreted as a means to increase individual signal efficiency. This contrasts with the situation prevailing in the barn owl in which siblings communicate vocally at a relatively low rate but for long periods of time. Similarly, in meerkats, Suricata suricatta, when pup-carers forage nearby, pups beg one after the other to avoid interference with littermates (Madden et al. 2009), a behaviour that increases total adult provisioning. In this situation, siblings can avoid calling simultaneously, which allows them to reduce their investment in vocal communication. The way siblings adjust call overlap is therefore an important and indicative component of sibling competition.

\section{Acknowledgments}

We thank Hélène Audusseau, Marine Battesti and Frederic Oberli for their assistance in the field. We are also grateful to Raphaelle Flint for useful comments. The study was financed by the Swiss National Science Foundation (SNF) to AR (grant no. 31003A_120517).

\section{References}

Aubin, T. \& Jouventin, P. 1998. Cocktail-party effect in king penguin colonies Proceedings of the Royal Society B, 265, 1665-1673.

Bee, M. A. \& Micheyl, C. 2008. The cocktail party problem. What is it? How can it be solved? And why should animal behaviorists study it? Journal of Comparative Psychology, 122, 235-251.

Blanc, A., Ogier, N., Roux, A., Denizeau, S. \& Mathevon, N. 2010. Begging coordination between siblings in black-headed gulls. Comptes Rendus Biologies, 333 688-693.

Boncoraglio, G., Caprioli, M. \& Saino, N. 2009. Fine-tuned modulation of competitive behaviour according to kinship in barn swallow nestlings. Proceedings of the Royal Society B, 276, 2117-2123.

Brumm, H. \& Slabbekoorn, H. 2005. Acoustic communication in noise. Advances in the Study of Behavior, 35, 151-209.

Bühler, P. \& Epple, W. 1980. Die Lautäußerungen der Schleiereule (Tyto alba). Journal of Ornithology, 121, 36-70.

Bulmer, E., Celis, P. \& Gil, D. 2008. Parent-absent begging: evidence for sibling honesty and cooperation in the spotless starling (Sturnus unicolor). Behaviora Ecology, 19, 279-284.

Carter, G. G., Skowronski, M. D., Faure, P. A. \& Fenton, B. 2008. Antiphonal calling allows individual discrimination in white-winged vampire bats. Animal Behaviour, 76, 1343-1355.

Chaiken, M. 1990. The ontogeny of antiphonal calling in European starlings. Developmental Psychobiology, 23, 233-246.

Chappell, M. A. \& Bachman, G. C. 2002. Energetic costs of begging behaviour. In: The Evolution of Begging: Competition, Cooperation and Communication (Ed. by J. Wright \& M. L. Leonard), pp. 143-162. Dordrecht: Kluwer Academic.

Dreiss, A. N., Henry, I., Ruppli, C., Almasi, B. \& Roulin, A. 2010a. Darker eumelanic barn owls better withstand food depletion through resistance to food deprivation and lower appetite. Oecologia, 164, 65-71.

Dreiss, A. N., Lahlah, N. \& Roulin, A. 2010b. How siblings adjust sib-sib communication and begging signals to each other. Animal Behaviour, 80, 1049-1055.

Dreiss, A. N., Ruppli, C. A., Faller, C. \& Roulin, A. 2013. Big brother is watching you: eavesdropping to resolve family conflicts. Behavioral Ecology, 24, 717-722.

Durant, J. M. \& Handrich, Y. 1998. Growth and food requirement flexibility in captive chicks of the European barn owl (Tyto alba). Journal of Zoology, 245, 137-145.

Ficken, R. W., Ficken, M. S. \& Hailman, J. P. 1974. Temporal pattern shifts to avoid acoustic interference in singing birds. Science, 183, 762-763.
Gerhardt, H. C. 1994. The evolution of vocalization in frogs and toads. Annual Review of Ecology and Systematics, 25, 293-324.

Godfray, H. C. J. 1995. Signaling of need between parents and young: parentoffspring conflict and sibling rivalry. American Naturalist, 146, 1-24.

Hamilton, W. D. 1964. The genetical evolution of social behaviour. Journal of Theoretical Biology, 7, 1-16.

Hultsch, H. \& Todt, D. 1982. Temporal performance roles during vocal interactions in nightingales (Luscinia megarhynchos B.). Behavioral Ecology and Sociobiology, 11, 253-260.

Johnstone, R. A. \& Roulin, A. 2003. Sibling negotiation. Behavioral Ecology, 14 780-786.

Kilner, R. M. 2001. A growth cost of begging in captive canary chicks. Proceedings of the National Academy of Sciences, U.S.A., 98, 11394-11398.

Kroodsma, D. E., Byers, B. E., Goodale, E., Johnson, S. \& Liu, W. C. 2001. Pseudoreplication in playback experiments, revisited a decade later. Animal Behaviour, 61, 1029-1033.

Leonard, M. L. \& Horn, A. G. 2001. Dynamics of calling by tree swallow (Tachycineta bicolor) nestmates. Behavioral Ecology and Sociobiology, 50, 430-435.

Madden, J. R., Kunc, H. P., English, S., Manser, M. B. \& Clutton-Brock, T. H. 2009 Calling in the gap: competition or cooperation in littermates' begging behaviour? Proceedings of the Royal Society B, 276, 1255-1262.

Magrath, R. D., Haff, T. M., Horn, A. G. \& Leonard, M. L. 2010. Calling in the face of danger: predation risk and acoustic communication by parent birds and their offspring. Advances in the Study of Behavior, 41, 187-253.

Maynard Smith, J. 1982. Do animals convey information about their intentions? Journal of Theoretical Biology, 97, 1e5.

Narins, P. M. \& Zelick, R. 1988. The effects of noise on auditory processing and behavior in amphibians. In: The Evolution of the Amphibian Auditory System (Ed. by B. Fritszch, W. Wilczynski, M. J. Ryan, T. Hetherington \& W. Walkowiak), pp. 511-536. New York: J. Wiley.

Parker, G. A. 1974. Assessment strategy and evolution of fighting behavior. Journal of Theoretical Biology, 47, 223-243.

Remage-Healey, L. \& Bass, A. H. 2006. From social behavior to neural circuitry: steroid hormones rapidly modulate advertisement calling via a vocal pattern generator. Hormones and Behavior, 50, 432-441.

Rodriguez-Gironés, M. A., Zuniga, J. M. \& Redondo, T. 2001. Effects of begging on growth rates of nestling chicks. Behavioral Ecology, 12, 269-274.

Römer, H. \& Bailey, W. 1998. Strategies for hearing in noise: peripheral control over auditory sensitivity in the bushcricket Sciarasaga quadrata (Austrosaginae: Tettigoniidae). Journal of Experimental Biology, 201, 1023-1033.

Roulin, A. 2002. The sibling negotiation hypothesis. In: The Evolution of Begging Competition, Cooperation and Communication (Ed. by J. Wright \& M. L. Leonard), pp. 107-127. Dordrecht: Kluwer Academic.

Roulin, A. 2004. Effects of hatching asynchrony on sibling negotiation, begging, jostling for position and within-brood food allocation in the barn owl, Tyto alba. Evolutionary Ecology Research, 6, 1083-1098.

Roulin, A., Kolliker, M. \& Richner, H. 2000. Barn owl (Tyto alba) siblings vocally negotiate resources. Proceedings of the Royal Society B, 267, 459-463.

Roulin, A., Muller, W., Sasvari, L., Dijkstra, C., Ducrest, A. L., Riols, C., Wink, M. \& Lubjuhn, T. 2004. Extra-pair paternity, testes size and testosterone level in relation to colour polymorphism in the barn owl Tyto alba. Journal of Avian Biology, 35, 492-500.

Roulin, A., Dreiss, A. N., Fioravanti, C. \& Bize, P. 2009. Vocal sib-sib interactions: how siblings adjust signalling level to each other. Animal Behaviour, 77, 717-725.

Schwartz, J. J. 1993. Male calling behavior, female discrimination and acoustic interference in the Neotropical treefrog Hyla microcephala under realistic conditions. Behavioral Ecology and Sociobiology, 32, 401-414.

Slabbekoorn, H. \& Peet, M. 2003. Birds sing at a higher pitch in urban noise: grea tits hit the high notes to ensure that their mating calls are heard above the city's din. Nature, 424, 267.

Smith, H. G. \& Montgomerie, R. 1991. Nestling American robins compete with siblings by begging. Behavioral Ecology and Sociobiology, 29, 307-312.

Stokes, A. W. \& Williams, H. W. 1968. Antiphonal calling in quail. Auk, 85, 83-89.

Todt, D. \& Naguib, M. 2000. Vocal interactions in birds: the use of song as a model in communication. Advances in the Study of Behavior, 29, 247-296.

Versace, E., Endress, A. D. \& Hauser, M. D. 2008. Pattern recognition mediates flexible timing of vocalizations in nonhuman primates: experiments with cottontop tamarins. Animal Behaviour, 76, 1885-1892.

Wright, J. \& Leonard, M. L. 2002. The Evolution of Begging: Competition, Cooperation and Communication. Dordrecht: Kluwer Academic. 